\title{
Fixed Point Result with Soft Cone Metric Space with Examples
}

\author{
Sneha A. Khandait ${ }^{(1)}$, Ramakant Bhardwaj ${ }^{(2)}$ and Chitra Singh ${ }^{(3)}$ \\ ${ }^{(1)}$ Research Scholar(Mathematics), Rabindranath Tagore University, Bhopal(M.P) \\ ${ }^{(2)}$ Department of Mathematics, Technocrats Institute of Technology, Bhopal (M.P.) \\ ${ }^{(3)}$ Department of Mathematics, Rabindranath Tagore University, Bhopal (M.P.)
}

\begin{abstract}
In the present paper, some fixed point and communal fixed point theorems are established for new contractive mapping in soft cone metric spaces. The generalized many previous well known results.
\end{abstract}

Keywords:soft cone metric space, communal fixed point theorem.

DOI: $10.7176 / \mathrm{MTM} / 9-4-07$

Publication date: April $30^{\text {th }} 2019$.

\section{Introduction:}

There are uncertainty in many complicated problems in the fields of engineering, physics, economics, computer science, medical science and social science. But to exceed these uncertainties some of some kind of theories were given like theory of fuzzy set [4], rough sets[2,3], theory of vague sets[1] i.e. which can use as mathematical tools for dealing with uncertainties. In 1999, Molodstov[5] initiated a novel concept of soft set theory as new mathematical toolfor dealing with uncertainty. Application of as soft set theory in other disciplines and real life problems are now catching momentum. Maji et al. [6,7] gave first practical application of soft sets in decision making problem. In the line of reduction and addition of parameters of soft sets, some work have been done by Chen et al. [8], Pei and Miao[9], Kong et al.[10]. Aktas[12] introduced a basic version of soft group theory. Shabir and Naz[13] studied soft topological spaces. Cetkin and Aygun [15] introduced the convergence of soft set net in soft topological spaces.Das and Samanta studied about soft real set and number[16], soft complex set and number[17], soft metric spaces[17,18], soft normed linear spaces[20,21]. Chiney and Samanta[22] introduced the notion of vector soft topology. Das et al. [23] studied on soft linear space and soft normed spaces.

In mathematical analysis the concept of communal fixed point theory in cone metric spaces is as common as banach's contraction principle is very important tool. In 2007, Huang and Zhang [25],introduced firstly cone metric space with normal cone generalization of metric spaces. Rezapour and Hamlbarani [26] gave the result of [25] for cone metric spaces without normality in cone. A lot of authors obtained common fixed point result for non-normal solid cones. There are many fixed point results that are concludeand performs different mapping about fixed point and communal fixed point which define by the concept of soft cone metric spaces. Ismet A. and Kemal T. [27] and M. Mohammad et al. [28] introduced the concept of soft cone metric spaces which is based on soft element. In this paper, we established some 
fixed point and communal fixed point theorems for new contractive condition in soft cone metric spaces. In rest of this section we recall some definitions with some examples which are needed in the sequel.

Definition 1.1. [28] Let (F,A) and (G,A) be two soft sets over a common universe E.

(i) (F,A) is said to be null soft set, denoted by $\emptyset$, if for all $\lambda \in A, F(\lambda)=\emptyset$. (F,A) is said to an absolute soft set denoted by $\tilde{E}$, if for all $\lambda \in \mathrm{A}, \mathrm{F}(\lambda)=\mathrm{E}$.

(ii) (F,A) is said to be a soft subset of $(\mathrm{G}, \mathrm{A})$ if for all $\lambda \in A, F(\lambda) \subseteq G(\lambda)$ and it is denoted by $(F, A) \widetilde{\subseteq}(G, A)$. (F,A) is said to be a soft upperset of $(G, A)$ if $(G, A)$ is a soft subset of $(F, A)$. We denote it by $(F, A) \supseteq(G, A)$. $(F, A)$ and $(G, A)$ is said to be equal if $(F, A)$ is a soft subset of $(\mathrm{G}, \mathrm{A})$ and $(\mathrm{G}, \mathrm{A})$ is a soft subset of $(\mathrm{F}, \mathrm{A})$.

(iii) The intersection $(H, A)$ of $(F, A)$ and $(G, A)$ over $E$ is defined as $H(\lambda)=F(\lambda) \cap G(\lambda)$ for all $\lambda \in \mathrm{A}$. We write $(\mathrm{F}, \mathrm{A}) \widetilde{\cap}(\mathrm{G}, \mathrm{A})=(\mathrm{H}, \mathrm{A})$.

(iv) The union $(H, A)$ of $(F, A)$ and $(G, A)$ over $E$ is defined as $H(\lambda)=F(\lambda) \cup G(\lambda)$ for all $\lambda \in A$. We write $(\mathrm{F}, \mathrm{A}) \widetilde{\mathrm{U}}(\mathrm{G}, \mathrm{A})=(\mathrm{H}, \mathrm{A})$.

(v) The cartesian product $(\mathrm{H}, \mathrm{A})$ of $(\mathrm{F}, \mathrm{A})$ and $(\mathrm{G}, \mathrm{A})$ over $\mathrm{E}$ denoted by $(\mathrm{H}, \mathrm{A})=(\mathrm{F}, \mathrm{A}) \widetilde{\mathrm{X}}(\mathrm{G}, \mathrm{A})$, is defined as $\mathrm{H}(\lambda)=\mathrm{F}(\lambda) \times \mathrm{G}(\lambda)$ for all $\lambda \in A$.

(vi) The difference $(\mathrm{H}, \mathrm{A})$ of $(\mathrm{F}, \mathrm{A})$ and $(\mathrm{G}, \mathrm{A})$ over $\mathrm{E}$ denoted by $(\mathrm{F}, \mathrm{A}) \tilde{/}(\mathrm{G}, \mathrm{A})=(\mathrm{H}, \mathrm{A})$, is defined as $H(\lambda)=F(\lambda) \backslash G(\lambda)$ for all $\lambda \in A$.

(vii) The complement of $(\mathrm{F}, \mathrm{A})$ is defined as $(\mathrm{F}, \mathrm{A})^{\mathrm{c}}=\left(\mathrm{F}^{\mathrm{c}}, \mathrm{A}\right)$, where $\mathrm{F}^{\mathrm{c}}: \mathrm{A} \rightarrow \mathrm{P}(\mathrm{E})$ is a mapping given by $\mathrm{F}^{\mathrm{c}}(\lambda)=\mathrm{B} \backslash \mathrm{F}(\lambda)$ for all $\lambda \in \mathrm{A}$. Clearly, we have $\widetilde{E^{c}}=\Phi$ and $\Phi^{\mathrm{c}}=\widetilde{E}$

Definition 1.2: [18] Let $X$ be a non-empty set and A be non-empty a parameter set. A mapping $\mathrm{d}: \operatorname{SE}(\tilde{X}) \times \operatorname{SE}(\tilde{X}) \rightarrow \mathrm{R}(\mathbb{A})^{*}$ is said to be a soft metric on the soft set $\tilde{X}$ if $\mathrm{d}$ satisfies the following conditions:

(i) $\mathrm{d}(\tilde{x}, \tilde{y}) \simeq \tilde{0}$, for all $, \tilde{x}, \tilde{y} \in \tilde{X}$.

(ii) $\mathrm{d}(\tilde{x}, \tilde{y})={ }^{-} 0$ if and only if $\tilde{x}=\tilde{y}$.

(iii) $\mathrm{d}(\tilde{x}, \tilde{y})=\mathrm{d}(\tilde{y}, \tilde{x})$ for all $\tilde{x}, \tilde{y} \widetilde{\in} \tilde{X}$.

(iv) $\mathrm{d}(\tilde{x}, \tilde{y}) \widetilde{\leq} \mathrm{d}(\tilde{x}, \tilde{y})+\mathrm{d}(\tilde{x}, \tilde{y})$ for all $\tilde{x}, \tilde{y}, \tilde{z} \widetilde{\in} \tilde{X}$.

The soft $\tilde{X}$ with a soft metric $\mathrm{d}$ on $\tilde{X}$ is said to be a soft metric space and denoted by $(\tilde{X}, \mathrm{~d}, \mathbb{A})$ or $(\tilde{X}, \mathrm{~d})$.

\section{Definition 1.3: [21,23]}

(a) A sequence $\left\{\widetilde{x_{n}}\right\}$ of soft elements in a soft normed linear space $(\tilde{X},\|\|,. \mathbb{A})$ is said to be convergent and converges to a soft element $\tilde{x}$ if $\left\|\widetilde{x_{n}}-\tilde{x}\right\| \rightarrow 0$ as $\mathrm{n} \rightarrow \infty$. This means for every $\tilde{\epsilon} \widetilde{>} \tilde{0}$, chosen arbitrarily, $\exists$ a natural number $N=N(\tilde{\epsilon})$ such that $\tilde{0} \widetilde{\leq}\left\|\widetilde{x_{n}}-\tilde{x}\right\| \widetilde{<} \tilde{\epsilon}$ whenever $n>N$ i.e. $n>N \Rightarrow \widetilde{x_{n}} \in B(\tilde{x}, \tilde{\epsilon}$ ), (where $B(\tilde{x}, \tilde{\epsilon})$ is an open ball with center $\tilde{x}$ and radius $\tilde{\epsilon}$ ). 
(b) A sequence $\left\{\widetilde{x_{n}}\right\}$ of soft elements in a soft normed linear space $(\tilde{X},\|\|,. \mathbb{A})$ is said to be a Cauchy sequence in $\tilde{X}$ if corresponding to every $\tilde{\epsilon} \widetilde{\nabla} \tilde{0} \exists$ a natural number $N=N(\tilde{\epsilon})$ such that $\left\|\widetilde{x_{n}}-\widetilde{x_{m}}\right\| \widetilde{\leq} \tilde{\epsilon}, \forall \mathrm{m}, \mathrm{n}>N$ i.e. $\left\|\widetilde{x_{n}}-\widetilde{x_{m}}\right\| \rightarrow \tilde{0}$ as $\mathrm{n}, \mathrm{m} \rightarrow \infty$.

(c) $\operatorname{Let}(\tilde{X},\|\|,. \mathbb{A})$ be a soft normed linear space. Then $\tilde{X}$ is said to be complete if every Cauchy sequence of soft elements in $\tilde{X}$ converges to a soft element of $\tilde{X}$. Every complete soft normed linear space is called a soft Banach space.

\section{SOFT CONE METRIC SPACES}

\section{Definition 1.4:[32]}

(a) Let $(\tilde{E},\|\|,. \mathbb{A})$ be a soft real Banach space and $(Q, \mathbb{A}) \in \mathrm{S}(\tilde{E})$ be a soft subset of $\tilde{E}$. Then $(Q, \mathbb{A})$ is called a soft cone if and only if

(1) $(Q, \mathbb{A})$ is closed, $(Q, \mathbb{A}) \neq \Phi$ and $(Q, \mathbb{A}) \neq \mathrm{SS}(\{\Theta\})$,

(2) $\tilde{a}, \tilde{b} \in \mathrm{R}(\mathbb{A})^{*}, \tilde{x}, \tilde{y} \in(\mathrm{Q}, \mathbb{A})$ implies $\tilde{a} \tilde{x}+\tilde{b} \tilde{y} \in(\mathrm{Q}, \mathbb{A})$,

(3) $\tilde{x} \widetilde{\epsilon}(\mathrm{Q}, \mathrm{A})$ and $-\tilde{x} \widetilde{\in}(\mathrm{Q}, \mathbb{A})$ implies $\tilde{x}=\Theta$.

Given a soft cone $(\mathrm{Q}, \mathbb{A}) \in \mathrm{S}(\tilde{E})$, we define a soft partial ordering $\preccurlyeq$ with respect to $(\mathrm{Q}, \mathbb{A})$ by $\tilde{x} \preccurlyeq \tilde{y}$ if and only if $\tilde{y}-\tilde{x} \in(\mathrm{Q}, \mathrm{A})$. We write $\tilde{x} \widetilde{y}$ to indicate that $\tilde{x} \preccurlyeq \tilde{y}$ but $\tilde{x} \neq \tilde{y}$, while $\tilde{x} \approx \tilde{y}$ will stand for $\tilde{y}-\tilde{x} \tilde{\in} \operatorname{Int}(\mathrm{Q}, \mathbb{A}), \operatorname{Int}(\mathrm{Q}, \mathbb{A})$ denotes the interior of $(\mathrm{Q}, \mathbb{A})$. The cone $\mathrm{Q}$ is known to be normal.

If there is constant $k>0$ such that for $\tilde{x}, \tilde{y} \in \tilde{E}, \Theta \preccurlyeq \tilde{x} \preccurlyeq \tilde{y}$ implies $\|\tilde{x}\| \preccurlyeq k\|\tilde{y}\|$.

(b)The least positive number satisfying this inequality is called the soft normal constant of $(\mathrm{Q}, \mathrm{A})$. The soft cone $(\mathrm{Q}, \mathbb{A})$ is called regular if every increasing sequence which is bounded from above is convergent. Equivalently the cone $(\mathrm{Q}, \mathrm{A})$ is called regular if every decreasing sequence which is bounded from below is convergent. Regular soft cones are soft normal and there exist soft normal cones which are not regular. Throughout the Banach space $\tilde{E}$ and the soft cone $(\mathrm{Q}, \mathrm{A})$ will be omitted.

Definition 1.5: [32] Let $X$ be a non-empty set and $\tilde{X}$ be absolute soft set. A mapping $\mathrm{d}$ : $\operatorname{SE}(\tilde{X}) \times \operatorname{SE}(\tilde{X}) \rightarrow \operatorname{SE}(\tilde{X})$ is said to be a soft cone metric on $\tilde{X}$ if d satisfies the following axioms:

i) $\Theta \widetilde{<} \mathrm{Q}(\tilde{x}, \tilde{y})$ for all $\tilde{x}, \tilde{y} \widetilde{\in} \tilde{X}$ and $\mathrm{Q}(\tilde{x}, \tilde{y})=\Theta$ iff $\tilde{x}=\tilde{y}$.

ii) $\mathrm{Q}(\tilde{x}, \tilde{y})=\mathrm{Q}(\tilde{y}, \tilde{x})$ for all $\tilde{x}, \tilde{y} \widetilde{\in} \tilde{X}$

iii) $\mathrm{Q}(\tilde{x}, \tilde{y}) \widetilde{\leq} \mathrm{Q}(\tilde{x}, \tilde{z})+\mathrm{Q}(\tilde{z}, \tilde{y})$ for all $\tilde{x}, \tilde{y}, \tilde{z} \widetilde{\in} \tilde{X}$

So,Then, the soft set $\tilde{X}$ with a soft cone metric $\mathrm{d}$ on $\tilde{X}$ is called a soft cone metric space and is denoted by $(\tilde{X}, \mathrm{Q}, \mathbb{A})$.

Then $\mathrm{Q}$ is called cone metric on $\tilde{X}$ and $(\tilde{X}, \mathrm{Q})$ is called soft cone metric space. 
Definition 1.6 : Suppose E and M be the mapping on a metric space $(\tilde{X}, Q)$ in a cone therefore it is said to be compatible if

$\lim _{n \rightarrow \infty} Q\left(E M \widetilde{x_{n}}, M E \widetilde{x_{n}}\right)=\Theta$

When the classification $\left\{\widetilde{x_{n}}\right\}$ in $\tilde{X}$ we have $\lim _{n \rightarrow \infty} E \widetilde{x_{n}}=\tilde{v}$ and $\lim _{n \rightarrow \infty} M \widetilde{x_{n}}=\tilde{v}$ for some $\tilde{v} \in \tilde{X}$. Let $\mathrm{E}$ and $\mathrm{M}$ be the identity mapping of the metric space $(\tilde{X}, \mathrm{Q})$ in cone therefore it is known as inadequately compatible, if the point of concurrence transform, then $E_{\tilde{\chi}}=M_{\tilde{\chi}}$ then implies that

$E M_{\tilde{x}}=M E_{\tilde{x}}$ for $\tilde{x} \in \tilde{X}$.

Example 1.7: let $\tilde{X}=\mathbb{C}_{\mathbb{R}}^{1}[0,1]$ with $\|\tilde{x}\|=\|\tilde{x}\|_{\infty}+\left\|x^{\prime}\right\|_{\infty}$

$$
(\mathrm{Q}, \mathbb{A})=\{\tilde{x} \in \tilde{X} \mid \tilde{x}(t) \cong \tilde{0}\}
$$

This cone is non-normal. Consider,

$\widetilde{x_{n}}(t)=\frac{t^{n+1}}{n+1}$ and $\widetilde{y_{n}}(t)=\frac{1}{n+1}$

Then , $\widetilde{0} \widetilde{\leq} \widetilde{x_{n}} \widetilde{\leq} \widetilde{y_{n}}$ and $\lim _{n \rightarrow \infty} \widetilde{y_{n}}=\tilde{0}$ but

$\left\|\widetilde{x_{n}}\right\|=\max \left\{t \in[0,1], \frac{t^{n+1}}{n+1}\right\}+\max \left\{t \in[0,1]: t^{n+1}\right\}$,

$$
\left\|\widetilde{x_{n}}\right\|=1
$$

Hence $\widetilde{x_{n}}$ does not converges to zero. This shows us sandwich theorem does not hold.

Example 1.8: let $\tilde{X}=\mathbb{C}_{\mathbb{R}}^{1}[0,1]$ with $\|\tilde{g}\|=\|\tilde{g}\|_{\infty}+\left\|\tilde{g}^{\prime}\right\|_{\infty}$

$$
(\mathrm{Q}, \mathbb{A})=\{\tilde{g} \in \tilde{X} \mid \tilde{g}(t) \geq \tilde{0}\}
$$

put

$\widetilde{x_{n}}(t)=\frac{1-\sin n t}{n}$ and $\widetilde{y_{n}}(t)=\frac{1+\sin n t}{n}$

Then, $\tilde{0} \widetilde{\leq} \widetilde{x_{n}} \widetilde{\leq} \widetilde{x_{n}}+\widetilde{y_{n}},\left\|\widetilde{x_{n}}\right\|=\left\|\widetilde{y_{n}}\right\|=1$

$\widetilde{x_{n}}+\widetilde{y_{n}}=\frac{2}{n} \rightarrow 0$.

The following examples verify some properties of definition 1.5

Example 1.9: Let $\mathbb{R}$ be all soft real number. Let $\mathbb{R}^{n}$ is soft banach space. Let $\tilde{X}=\mathbb{R}^{n}$ with

$$
(\mathrm{Q}, \mathrm{A})=\operatorname{SS}\left\{\left(\widetilde{x_{1}}, \widetilde{x_{2}}, \ldots \widetilde{x_{n}}\right): \widetilde{x_{l}} \geq \tilde{0}, \forall i=1,2, \ldots n\right\}
$$

The soft cone $(\mathrm{Q}, \mathbb{A})$ is normal, generating, minihedral, strongly minihedral and solid.

Example 1.10:Let with $k \sim 1$ be given. Consider the real vector space with

$\tilde{X}=\left\{\tilde{c} \tilde{x}+\tilde{d}: \tilde{c}, \tilde{d} \widetilde{\in} \mathbb{R} ; \tilde{x} \widetilde{\in}\left[1-\frac{1}{k}, 1\right]\right\}$ 
With the supremum norm and soft cone

$$
(\mathrm{Q}, \mathbb{A})=\{\tilde{c} \tilde{x}+\tilde{d} ; \tilde{c} \simeq \tilde{0}, \tilde{d} \widetilde{\leq} \tilde{0}\}
$$

in $\tilde{X}$. The soft cone $(\mathrm{Q}, \mathbb{A})$ is regular and so normal.

Example 1.11:Let $\tilde{X}=\mathbb{R}^{2}$ and

$$
(\mathrm{Q}, \mathbb{A})=\{(\tilde{x}, \tilde{y}): \tilde{x}, \tilde{y} \geq \tilde{0}\}
$$

The cone $(\mathrm{Q}, \mathbb{A})$ is strongly minihedral in which each subset of $(\mathrm{Q}, \mathbb{A})$ has infimum.

Example 1.12: Let $\tilde{X}=\mathbb{R}^{2}$ and

$(\mathrm{Q}, \mathbb{A})=\{(\tilde{x}, \tilde{0}): \tilde{x} \simeq \tilde{0}\}$

This $(\mathrm{Q}, \mathrm{A})$ is strongly minihedral but not minihedral.

Example 1.13:Let $\tilde{X}$ be real vector space,

$\tilde{X}=\left\{\tilde{c} \tilde{x}+\tilde{d} ; \tilde{c}, \tilde{d} \in \mathbb{R} ; \tilde{x} \in\left[\frac{2}{3}, 1\right]\right\}$

With the supremum norm and

$(\mathrm{Q}, \mathbb{A})=\{\tilde{c} \tilde{x}+\tilde{d} ; \tilde{c} \widetilde{\leq} \tilde{0}, \tilde{d} \cong \tilde{0}\}$

So, $(\mathrm{Q}, \mathrm{A})$ is normal soft cone in $\tilde{X}$ with constant $\tilde{k}>1$.Define

$f(\tilde{x})=-6 \tilde{x}+13, g(\tilde{x})=-9 \tilde{x}+14 \in(Q, \mathbb{A})$

Then $f \leq g$ as $g(\tilde{x})-f(\tilde{x})=-3 \tilde{x}+1 \in(Q, \mathbb{A})$.

But

$$
\|f\|=f\left(\frac{2}{3}\right)=9, \quad\|g\|=g\left(\frac{2}{3}\right)=8
$$

Therefore, $f \leq g$ but $\|f\| \geq\|g\|$.

\section{Main Results:}

Theorem 2.1: Let the metric space $(\tilde{X}, \mathrm{~d})$ be with complete cone has $(\mathrm{Q}, \mathbb{A})$ is regular through $\mathrm{r}$ as regular constant. Supposing the map representing A from $\tilde{X}$ into itself satisfies the condition

$$
\begin{aligned}
d(A \tilde{x}, A \tilde{y}) \leq & \alpha d(\tilde{x}, \tilde{y})+\beta[d(\tilde{x}, A \tilde{x})+d(\tilde{y}, A \tilde{y})+\gamma[d(\tilde{x}, A \tilde{y})+d(\tilde{y}, A \tilde{x})] \\
+e \max \{d(\tilde{x}, A \tilde{x})+ & d(\tilde{y}, A \tilde{y})\}+h \max \{d(\tilde{x}, A \tilde{y}), d(\tilde{y}, A \tilde{x})\} \\
& +\delta\left[\frac{d(\tilde{x}, A \tilde{x})+d(\tilde{y}, A \tilde{y})+d(\tilde{x}, A \tilde{y})+d(\tilde{y}, A \tilde{x})}{1+d(\tilde{x}, A \tilde{x}) d(\tilde{y}, A \tilde{y}) d(\tilde{x}, A \tilde{y}) d(\tilde{y}, A \tilde{x})}\right]
\end{aligned}
$$


For every $\tilde{x}, \tilde{y} \widetilde{\in} \tilde{X}$ and $\alpha, \beta, \gamma, e, h, \delta \geq 0$ such that $0 \leq(\beta+\gamma+h+2 \delta)+\left(\frac{\alpha+e}{2}\right)<\frac{1}{2}$. Then A has communal invariant point in $\tilde{X}$.

Proof:For any chance $\widetilde{x_{0}}$ in $\tilde{X}$, we have to choose $\widetilde{x_{1}}, \widetilde{x_{2}} \in \tilde{X}$ such that $A \widetilde{x_{0}}=\widetilde{x_{1}}$ and $A \widetilde{x_{1}}=\widetilde{x_{2}}$

Also, in general we can define a classification of elements in $\mathrm{X}$ we have $\widetilde{x_{2 n+1}}=A \widetilde{x_{2 n}}$ and $\widetilde{x_{2 n+2}}=A \widetilde{x_{2 n+1}}$

Now, $d\left(\widetilde{x_{2 n+1}}, \widetilde{x_{2 n+2}}\right)=d\left(\widetilde{x_{2 n}}, A \widetilde{x_{2 n+1}}\right)$

From [2.1.1]

$$
\begin{aligned}
& d\left(A \widetilde{x_{2 n}}, A \widetilde{x_{2 n+1}}\right) \\
& \leq \alpha d\left(\widetilde{x_{2 n}}, \widetilde{x_{2 n+1}}\right)+\beta\left[d\left(\widetilde{x_{2 n}}, A \widetilde{x_{2 n}}\right)+d\left(\widetilde{x_{2 n+1}}, A \widetilde{x_{2 n+1}}\right)\right]+ \\
& \gamma\left[d\left(\widetilde{x_{2 n}}, A \widetilde{x_{2 n+1}}\right)+d\left(\widetilde{x_{2 n+1}}, A \widetilde{x_{2 n}}\right)\right]+e \max \left\{d\left(\widetilde{x_{2 n}}, A \widetilde{x_{2 n}}\right), d\left(\widetilde{x_{2 n+1}}, A \widetilde{x_{2 n+1}}\right)\right\} \\
& +h \max \left\{d\left(\widetilde{x_{2 n}}, A \widetilde{x_{2 n+1}}\right), d\left(\widetilde{x_{2 n+1}}, A \widetilde{x_{2 n}}\right)\right\}+ \\
& \delta \frac{\left[d\left(\widetilde{x_{2 n}}, A \widetilde{x_{2 n}}\right)+d\left(\widetilde{x_{2 n+1}}, A \widetilde{x_{2 n+1}}\right)+d\left(\widetilde{x_{2 n}}, A \widetilde{x_{2 n+1}}\right)+d\left(\widetilde{x_{2 n+1}}, A \widetilde{x_{2 n}}\right)\right]}{1+d\left(\widetilde{x_{2 n}}, A \widetilde{x_{2 n}}\right) d\left(\widetilde{x_{2 n+1}}, A \widetilde{x_{2 n+1}}\right) d\left(\widetilde{x_{2 n}}, A \widetilde{x_{2 n+1}}\right) d\left(\widetilde{x_{2 n+1}}, A \widetilde{x_{2 n}}\right)} \\
& d\left(\widetilde{x_{2 n+1}}, \widetilde{x_{2 n+2}}\right) \\
& \leq \alpha d\left(\widetilde{x_{2 n}}, \widetilde{x_{2 n+1}}\right)+\beta\left[d\left(\widetilde{x_{2 n}}, \widetilde{x_{2 n+1}}\right)+d\left(\widetilde{x_{2 n+1}}, \widetilde{x_{2 n+2}}\right)\right]+\gamma\left[d\left(\widetilde{x_{2 n}}, \widetilde{x_{2 n+2}}\right)\right] \\
& +e \max \left\{d\left(\widetilde{x_{2 n}}, \widetilde{x_{2 n+1}}\right), d\left(\widetilde{x_{2 n+1}}, \widetilde{x_{2 n+2}}\right)\right\}+h \max \left\{d\left(\widetilde{x_{2 n}}, \widetilde{x_{2 n+2}}\right)\right\} \\
& +\delta \frac{\left[d\left(\widetilde{x_{2 n}}, \widetilde{x_{2 n+1}}\right)+d\left(\widetilde{x_{2 n+1}}, \widetilde{x_{2 n+2}}\right)+d\left(\widetilde{x_{2 n},} \widetilde{x_{2 n+2}}\right)+d\left(\widetilde{x_{2 n+1}}, \widetilde{x_{2 n+1}}\right)\right]}{1+d\left(\widetilde{x_{2 n}}, \widetilde{x_{2 n+1}}\right) d\left(\widetilde{x_{2 n+1}, \widetilde{x_{2 n+2}}}\right) d\left(\widetilde{x_{2 n}}, \widetilde{x_{2 n+2}}\right) d\left(\widetilde{x_{2 n+1}}, \widetilde{x_{2 n+1}}\right)}
\end{aligned}
$$

\section{Consider case I:}

$$
\begin{gathered}
\operatorname{Let} d\left(\widetilde{x_{2 n}}, \widetilde{x_{2 n+1}}\right)>d\left(\widetilde{x_{2 n+1}}, \widetilde{x_{2 n+2}}\right) \\
\text { So, } \max \left\{d\left(\widetilde{x_{2 n}}, \widetilde{x_{2 n+1}}\right), d\left(\widetilde{x_{2 n+1}}, \widetilde{x_{2 n+2}}\right)\right\}=d\left(\widetilde{x_{2 n}}, \widetilde{x_{2 n+1}}\right) \\
d\left(\widetilde{x_{2 n+1}}, \widetilde{x_{2 n+2}}\right) \\
\leq \alpha d\left(\widetilde{x_{2 n}}, \widetilde{x_{2 n+1}}\right)+\beta\left[d\left(\widetilde{x_{2 n}}, \widetilde{x_{2 n+1}}\right)+d\left(\widetilde{x_{2 n+1}}, \widetilde{x_{2 n+2}}\right)\right]+\gamma\left[d\left(\widetilde{x_{2 n}}, \widetilde{x_{2 n+2}}\right)\right] \\
+e d\left(\widetilde{x_{2 n}}, \widetilde{x_{2 n+1}}\right)+h \max \left\{d\left(\widetilde{x_{2 n}}, \widetilde{x_{2 n+2}}\right)\right\}+2 \delta\left[d\left(\widetilde{x_{2 n}}, \widetilde{x_{2 n+1}}\right)+d\left(\widetilde{x_{2 n+1}}, \widetilde{x_{2 n+2}}\right)\right] \\
\leq(\alpha+\beta+\gamma+e+h+2 \delta) d\left(\widetilde{x_{2 n}}, \widetilde{x_{2 n+1}}\right)+(\beta+\gamma+h+2 \delta) d\left(\widetilde{x_{2 n+1}}, \widetilde{x_{2 n+2}}\right) \\
(1-\beta-\gamma-h-2 \delta) d\left(\widetilde{x_{2 n+1}}, \widetilde{x_{2 n+2}}\right) \leq(\alpha+\beta+\gamma+e+h+2 \delta) d\left(\widetilde{x_{2 n}}, \widetilde{x_{2 n+1}}\right) \\
d\left(\widetilde{x_{2 n+1}}, \widetilde{x_{2 n+2}}\right) \leq \frac{(\alpha+\beta+\gamma+e+h+2 \delta)}{(1-\beta-\gamma-h-2 \delta)} d\left(\widetilde{x_{2 n}}, \widetilde{x_{2 n+1}}\right)
\end{gathered}
$$

Simillaraly we can show that 


$$
d\left(\widetilde{x_{2 n}}, \widetilde{x_{2 n+1}}\right) \leq \frac{(\alpha+\beta+\gamma+e+h+2 \delta)}{(1-\beta-\gamma-h-2 \delta)} d\left(\widetilde{x_{2 n-1}}, \widetilde{x_{2 n}}\right)
$$

In general we can write,

$$
d\left(\widetilde{x_{2 n+1}}, \widetilde{x_{2 n+2}}\right)=\left[\frac{(\alpha+\beta+\gamma+e+h+2 \delta)}{(1-\beta-\gamma-h-2 \delta)}\right]^{2 n+1} d\left(\widetilde{x_{0}}, \widetilde{x_{1}}\right)
$$

On taking $\frac{(\alpha+\beta+\gamma+e+h+2 \delta)}{(1-\beta-\gamma-h-2 \delta)}=\phi_{1}$

$$
d\left(\widetilde{x_{2 n+1}}, \widetilde{x_{2 n+2}}\right) \leq \phi_{1}{ }^{2 n+1} d\left(\widetilde{x_{0}}, \widetilde{x_{1}}\right)
$$

\section{Case II:}

Let $d\left(\widetilde{x_{2 n+1}}, \widetilde{x_{2 n+2}}\right)>d\left(\widetilde{x_{2 n}}, \widetilde{x_{2 n+1}}\right)$

$$
\begin{gathered}
\text { So, } \max \left\{d\left(\widetilde{x_{2 n}}, \widetilde{x_{2 n+1}}\right), d\left(\widetilde{x_{2 n+1}}, \widetilde{x_{2 n+2}}\right)\right\}=d\left(\widetilde{x_{2 n+1}}, \widetilde{x_{2 n+2}}\right) \\
d\left(\widetilde{x_{2 n+1}}, \widetilde{x_{2 n+2}}\right) \\
\leq \alpha d\left(\widetilde{x_{2 n}}, \widetilde{x_{2 n+1}}\right)+\beta\left[d\left(\widetilde{x_{2 n}}, \widetilde{x_{2 n+1}}\right)+d\left(\widetilde{x_{2 n+1}}, \widetilde{x_{2 n+2}}\right)\right]+\gamma\left[d\left(\widetilde{x_{2 n}}, \widetilde{x_{2 n+2}}\right)\right] \\
+e d\left(\widetilde{x_{2 n+1}}, \widetilde{x_{2 n+2}}\right)+h \max \left\{d\left(\widetilde{x_{2 n}}, \widetilde{x_{2 n+2}}\right)\right\}+2 \delta\left[d\left(\widetilde{x_{2 n}}, \widetilde{x_{2 n+1}}\right)+d\left(\widetilde{x_{2 n+1}}, \widetilde{x_{2 n+2}}\right)\right] \\
\leq(\alpha+\beta+\gamma+h+2 \delta) d\left(\widetilde{x_{2 n}}, \widetilde{x_{2 n+1}}\right)+(\beta+\gamma+e+h+2 \delta) d\left(\widetilde{x_{2 n+1}}, \widetilde{x_{2 n+2}}\right) \\
(1-\beta-\gamma-e-h-2 \delta) d\left(\widetilde{x_{2 n+1}}, \widetilde{x_{2 n+2}}\right) \leq(\alpha+\beta+\gamma+h+2 \delta) d\left(\widetilde{x_{2 n}}, \widetilde{x_{2 n+1}}\right) \\
d\left(\widetilde{x_{2 n+1}}, \widetilde{x_{2 n+2}}\right) \leq \frac{(\alpha+\beta+\gamma+h+2 \delta)}{(1-\beta-\gamma-e-h-2 \delta)} d\left(\widetilde{x_{2 n}}, \widetilde{x_{2 n+1}}\right)
\end{gathered}
$$

Simillaraly we can show that

$$
d\left(\widetilde{x_{2 n}}, \widetilde{x_{2 n+1}}\right) \leq \frac{(\alpha+\beta+\gamma+h+2 \delta)}{(1-\beta-\gamma-e-h-2 \delta)} d\left(\widetilde{x_{2 n-1}}, \widetilde{x_{2 n}}\right)
$$

In general we can write,

$$
d\left(\widetilde{x_{2 n+1}}, \widetilde{x_{2 n+2}}\right)=\left[\frac{(\alpha+\beta+\gamma+h+2 \delta)}{(1-\beta-\gamma-e-h-2 \delta)}\right]^{2 n+1} d\left(\widetilde{x_{0}}, \widetilde{x_{1}}\right)
$$

On taking $\frac{(\alpha+\beta+\gamma+h+2 \delta)}{(1-\beta-\gamma-e-h-2 \delta)}=\phi_{2}$

$$
d\left(\widetilde{x_{2 n+1}}, \widetilde{x_{2 n+2}}\right) \leq \phi_{2}{ }^{2 n+1} d\left(\widetilde{x_{0}}, \widetilde{x_{1}}\right)
$$

Let, $\phi=\max \left\{\phi_{1}, \phi_{2}\right\}$

For $n \leq m$ we have

$$
\begin{aligned}
& d\left(\widetilde{x_{2 n}}, \widetilde{x_{2 m}}\right) \leq d\left(\widetilde{x_{2 n}}, \widetilde{x_{2 n+1}}\right)+d\left(\widetilde{x_{2 n+1}}, \widetilde{x_{2 n+2}}\right)+\ldots \ldots \ldots \ldots+d\left(\widetilde{x_{2 m-1}}, \widetilde{x_{2 m}}\right) \\
& \leq\left(\phi^{n}+\phi^{n+1}+\phi^{n+2}+\ldots \ldots+\phi^{m}\right) d\left(\widetilde{x_{0}}, \widetilde{x_{1}}\right)
\end{aligned}
$$




$$
d\left(\widetilde{x_{2 n}}, \widetilde{x_{2 m}}\right) \leq \frac{\phi^{n}}{1-\phi} d\left(\widetilde{x_{0}}, \widetilde{x_{1}}\right)
$$

$\left\|d\left(\widetilde{x_{2 n}}, \widetilde{x_{2 m}}\right)\right\| \leq \frac{\phi^{n}}{1-\phi} r\left\|d\left(\widetilde{x_{0}}, \widetilde{x_{1}}\right)\right\|$ as $n \rightarrow \infty$

$$
\lim _{n \rightarrow \infty}\left\|d\left(\widetilde{x_{2 n}}, \widetilde{x_{2 m}}\right)\right\| \rightarrow 0
$$

Hence $\left\{\widetilde{x_{n}}\right\}$ is Cauchy classification in which $\tilde{v}$ touches to $\tilde{X}$.

Hence a metric $(\tilde{X}, \mathrm{~d})$ space is complete soft cone. Therefore for $\widetilde{x_{n}} \rightarrow \tilde{v}$ as $n \rightarrow \infty, A \widetilde{x_{n}} \rightarrow$ $\tilde{v}$ as $n \rightarrow \infty$.

We have $\tilde{v}$ is an invariant point of $\mathrm{A}$ in $\tilde{X}$.

Uniqueness:Let us suppose that there is another invariant point of A, i.e. $\tilde{v}$ in $\tilde{X}$ which is distinct from $\tilde{v}$, then

$$
\begin{aligned}
& A \widetilde{w}=\widetilde{w} \text { and } A \tilde{v}=\tilde{v} \\
& d(\tilde{v}, \widetilde{w})=d(A \tilde{v}, A \widetilde{w})
\end{aligned}
$$

From [2.1.1]

$$
\begin{aligned}
& d(A \tilde{v}, A \widetilde{w}) \leq \alpha d(\tilde{v}, \widetilde{w})+\beta[d(\tilde{v}, A \tilde{v})+d(\widetilde{w}, A \widetilde{w})]+\gamma[d(\widetilde{v}, A \widetilde{w})+d(\widetilde{w}, A \tilde{v})]+ \\
& e \max \{d(\widetilde{v}, A \tilde{v}), d(\widetilde{w}, A \widetilde{w})\}+h \max \{d(\widetilde{v}, A \widetilde{w}), d(\widetilde{w}, A \widetilde{v})+ \\
& \delta\left[\frac{d(\tilde{v}, A \tilde{v})+d(\widetilde{w}, A \widetilde{w})+d(\tilde{v}, A \widetilde{w})+d(\widetilde{w}, A \tilde{v})}{1+d(\tilde{v}, A \tilde{v}) d(\widetilde{w}, A \widetilde{w}) d(\tilde{v}, A \widetilde{w}) d(\widetilde{w}, A \tilde{v})}\right] \\
& \leq \alpha d(\tilde{v}, \widetilde{w})+\beta .0+2 \gamma d(\tilde{v}, \widetilde{w})+h \max \{d(\widetilde{v}, \widetilde{w}), d(\widetilde{w}, \tilde{v})\}+2 \delta d(\tilde{v}, \widetilde{w}) \\
& \leq \alpha d(\tilde{v}, \widetilde{w})+2 \gamma d(\tilde{v}, \widetilde{w})+h d(\tilde{v}, \widetilde{w})+2 \delta d(\widetilde{v}, \widetilde{w}) \\
& d(A \tilde{v}, A \widetilde{w}) \leq(\alpha+2 \gamma+h+2 \delta) d(\tilde{v}, \widetilde{w})
\end{aligned}
$$

Which gives a contradiction. Thus $\tilde{v}$ is an communal fixed point of $\mathrm{A}$ in $\tilde{X}$.

Theorem 2.2:Let the metric space $(\tilde{X}, \mathrm{~d})$ be with complete soft cone has $(\mathrm{Q}, \mathbb{A})$ is regular through $r$ as regular constant. Let the map representing A, B from $\tilde{X}$ into itself satisfies the condition

$$
\begin{aligned}
& \quad d(A \tilde{x}, B \tilde{y}) \leq \alpha d(\tilde{x}, \tilde{y})+\beta[d(\tilde{x}, A \tilde{x})+d(\tilde{y}, B \tilde{y})+\gamma[d(\tilde{x}, B \tilde{y})+d(\tilde{y}, A \tilde{x})] \\
& +e \max \{d(\tilde{x}, A \tilde{x})+d(\tilde{y}, B \tilde{y})\}+h \max \{d(\tilde{x}, B \tilde{y}), d(\tilde{y}, A \tilde{x})\} \\
& +\delta\left[\frac{d(\tilde{x}, A \tilde{x})+d(\tilde{y}, B \tilde{y})+d(\tilde{x}, B \tilde{y})+d(\tilde{y}, A \tilde{x})}{1+d(\tilde{x} A \tilde{x}) d(\tilde{y}, B \tilde{y}) d(\tilde{x}, B \tilde{y}) d(\tilde{y}, A \tilde{x})}\right]
\end{aligned}
$$

For every $\tilde{x}, \tilde{y} \widetilde{\in} \tilde{X}$ and $\alpha, \beta, \gamma, e, h, \delta \geq 0$ such that $0 \leq(\beta+\gamma+h+2 \delta)+\left(\frac{\alpha+e}{2}\right)<\frac{1}{2}$. Then A, B has communal invariant point in $\tilde{X}$. 
Proof:For any chance $\widetilde{x_{0}}$ in $\tilde{X}$, we have to choose $\widetilde{x_{1}}, \widetilde{x_{2}} \in \tilde{X}$ such that

$A \widetilde{x_{0}}=\widetilde{x_{1}}$ and $B \widetilde{x_{1}}=\widetilde{x_{2}}$

Also, in general we can define a classification of elements in $\mathrm{X}$ we have

$\widetilde{x_{2 n+1}}=A \widetilde{x_{2 n}}$ and $\widetilde{x_{2 n+2}}=B \widetilde{x_{2 n+1}}$

Now, $d\left(\widetilde{x_{2 n+1}}, \widetilde{x_{2 n+2}}\right)=d\left(A \widetilde{x_{2 n}}, B \widetilde{x_{2 n+1}}\right)$

From [2.2.1]

$$
\begin{aligned}
& d\left(A \widetilde{x_{2 n}}, B \widetilde{x_{2 n+1}}\right) \\
& \leq \alpha d\left(\widetilde{x_{2 n}}, \widetilde{x_{2 n+1}}\right)+\beta\left[d\left(\widetilde{x_{2 n}}, A \widetilde{x_{2 n}}\right)+d\left(\widetilde{x_{2 n+1}}, B \widetilde{x_{2 n+1}}\right)\right]+ \\
& \gamma\left[d\left(\widetilde{x_{2 n}}, B \widetilde{x_{2 n+1}}\right)+d\left(\widetilde{x_{2 n+1}}, A \widetilde{x_{2 n}}\right)\right]+e \max \left\{d\left(\widetilde{x_{2 n}}, A \widetilde{x_{2 n}}\right), d\left(\widetilde{x_{2 n+1}}, B \widetilde{x_{2 n+1}}\right)\right\} \\
& +h \max \left\{d\left(\widetilde{x_{2 n}}, B \widetilde{x_{2 n+1}}\right), d\left(\widetilde{x_{2 n+1}}, A \widetilde{x_{2 n}}\right)\right\}+ \\
& \delta \frac{\left[d\left(\widetilde{x_{2 n}}, A \widetilde{x_{2 n}}\right)+d\left(\widetilde{x_{2 n+1}}, B \widetilde{x_{2 n+1}}\right)+d\left(\widetilde{x_{2 n}}, B \widetilde{x_{2 n+1}}\right)+d\left(\widetilde{x_{2 n+1}}, A \widetilde{x_{2 n}}\right)\right]}{1+d\left(\widetilde{x_{2 n}}, A \widetilde{x_{2 n}}\right) d\left(\widetilde{x_{2 n+1}}, B \widetilde{x_{2 n+1}}\right) d\left(\widetilde{x_{2 n}}, B \widetilde{x_{2 n+1}}\right) d\left(\widetilde{x_{2 n+1}}, A \widetilde{x_{2 n}}\right)} \\
& d\left(\widetilde{x_{2 n+1}}, \widetilde{x_{2 n+2}}\right) \\
& \leq \alpha d\left(\widetilde{x_{2 n}}, \widetilde{x_{2 n+1}}\right)+\beta\left[d\left(\widetilde{x_{2 n}}, \widetilde{x_{2 n+1}}\right)+d\left(\widetilde{x_{2 n+1}}, \widetilde{x_{2 n+2}}\right)\right]+\gamma\left[d\left(\widetilde{x_{2 n}}, \widetilde{x_{2 n+2}}\right)\right] \\
& +e \max \left\{d\left(\widetilde{x_{2 n}}, \widetilde{x_{2 n+1}}\right), d\left(\widetilde{x_{2 n+1}}, \widetilde{x_{2 n+2}}\right)\right\}+h\left\{d\left(\widetilde{x_{2 n}}, \widetilde{x_{2 n+2}}\right)\right\} \\
& +\delta \frac{\left[d\left(\widetilde{x_{2 n}}, \widetilde{x_{2 n+1}}\right)+d\left(\widetilde{x_{2 n+1}}, \widetilde{x_{2 n+2}}\right)+d\left(\widetilde{x_{2 n},} \widetilde{x_{2 n+2}}\right)+d\left(\widetilde{x_{2 n+1}}, \widetilde{x} \widetilde{x_{2 n+1}}\right)\right]}{1+d\left(\widetilde{x_{2 n+1}}\right) d\left(\widetilde{x_{2 n+1}, \widetilde{x_{2 n+2}}}\right) d\left(\widetilde{x_{2 n},}, \widetilde{x_{2 n+2}}\right) d\left(\widetilde{x_{2 n+1},}, \widetilde{x_{2 n+1}}\right)}
\end{aligned}
$$

\section{Consider case I:}

$$
\begin{gathered}
\text { Let } d\left(\widetilde{x_{2 n}}, \widetilde{x_{2 n+1}}\right)>d\left(\widetilde{x_{2 n+1}}, \widetilde{x_{2 n+2}}\right) \\
\text { So, } \max \left\{d\left(\widetilde{x_{2 n}}, \widetilde{x_{2 n+1}}\right), d\left(\widetilde{x_{2 n+1}}, \widetilde{x_{2 n+2}}\right)\right\}=d\left(\widetilde{x_{2 n}}, \widetilde{x_{2 n+1}}\right) \\
d\left(\widetilde{x_{2 n+1}}, \widetilde{x_{2 n+2}}\right) \\
\leq \alpha d\left(\widetilde{x_{2 n}}, \widetilde{x_{2 n+1}}\right)+\beta\left[d\left(\widetilde{x_{2 n}}, \widetilde{x_{2 n+1}}\right)+d\left(\widetilde{x_{2 n+1}}, \widetilde{x_{2 n+2}}\right)\right]+\gamma\left[d\left(\widetilde{x_{2 n}}, \widetilde{x_{2 n+2}}\right)\right] \\
+e d\left(\widetilde{x_{2 n}}, \widetilde{x_{2 n+1}}\right)+h \max \left\{d\left(\widetilde{x_{2 n}}, \widetilde{x_{2 n+2}}\right)\right\}+2 \delta\left[d\left(\widetilde{x_{2 n}}, \widetilde{x_{2 n+1}}\right)+d\left(\widetilde{x_{2 n+1}}, \widetilde{x_{2 n+2}}\right)\right] \\
\leq(\alpha+\beta+\gamma+e+h+2 \delta) d\left(\widetilde{x_{2 n}}, \widetilde{x_{2 n+1}}\right)+(\beta+\gamma+h+2 \delta) d\left(\widetilde{x_{2 n+1}}, \widetilde{x_{2 n+2}}\right) \\
(1-\beta-\gamma-h-2 \delta) d\left(\widetilde{x_{2 n+1}}, \widetilde{x_{2 n+2}}\right) \leq(\alpha+\beta+\gamma+e+h+2 \delta) d\left(\widetilde{x_{2 n}}, \widetilde{x_{2 n+1}}\right) \\
d\left(\widetilde{x_{2 n+1}}, \widetilde{x_{2 n+2}}\right) \leq \frac{(\alpha+\beta+\gamma+e+h+2 \delta)}{(1-\beta-\gamma-h-2 \delta)} d\left(\widetilde{x_{2 n}}, \widetilde{x_{2 n+1}}\right)
\end{gathered}
$$

Similarly we can show that

$$
d\left(\widetilde{x_{2 n}}, \widetilde{x_{2 n+1}}\right) \leq \frac{(\alpha+\beta+\gamma+e+h+2 \delta)}{(1-\beta-\gamma-h-2 \delta)} d\left(\widetilde{x_{2 n-1}}, \widetilde{x_{2 n}}\right)
$$

In general we can write, 


$$
d\left(\widetilde{x_{2 n+1}}, \widetilde{x_{2 n+2}}\right)=\left[\frac{(\alpha+\beta+\gamma+e+h+2 \delta)}{(1-\beta-\gamma-h-2 \delta)}\right]^{2 n+1} d\left(\widetilde{x_{0}}, \widetilde{x_{1}}\right)
$$

On taking $\frac{(\alpha+\beta+\gamma+e+h+2 \delta)}{(1-\beta-\gamma-h-2 \delta)}=\phi_{1}$

$$
d\left(\widetilde{x_{2 n+1}}, \widetilde{x_{2 n+2}}\right) \leq \phi_{1}{ }^{2 n+1} d\left(\widetilde{x_{0}}, \widetilde{x_{1}}\right)
$$

\section{Case II:}

Let $d\left(\widetilde{x_{2 n+1}}, \widetilde{x_{2 n+2}}\right)>d\left(\widetilde{x_{2 n}}, \widetilde{x_{2 n+1}}\right)$

So, $\max \left\{d\left(\widetilde{x_{2 n}}, \widetilde{x_{2 n+1}}\right), d\left(\widetilde{x_{2 n+1}}, \widetilde{x_{2 n+2}}\right)\right\}=d\left(\widetilde{x_{2 n+1}}, \widetilde{x_{2 n+2}}\right)$

$$
d\left(\widetilde{x_{2 n+1}}, \widetilde{x_{2 n+2}}\right)
$$

$\leq \alpha d\left(\widetilde{x_{2 n}}, \widetilde{x_{2 n+1}}\right)+\beta\left[d\left(\widetilde{x_{2 n}}, \widetilde{x_{2 n+1}}\right)+d\left(\widetilde{x_{2 n+1}}, \widetilde{x_{2 n+2}}\right)\right]+\gamma\left[d\left(\widetilde{x_{2 n}}, \widetilde{x_{2 n+2}}\right)\right]$

$+e d\left(\widetilde{x_{2 n+1}}, \widetilde{x_{2 n+2}}\right)+h \max \left\{d\left(\widetilde{x_{2 n}}, \widetilde{x_{2 n+2}}\right)\right\}+2 \delta\left[d\left(\widetilde{x_{2 n}}, \widetilde{x_{2 n+1}}\right)+d\left(\widetilde{x_{2 n+1}}, \widetilde{x_{2 n+2}}\right)\right]$

$$
\begin{gathered}
\leq(\alpha+\beta+\gamma+h+2 \delta) d\left(\widetilde{x_{2 n}}, \widetilde{x_{2 n+1}}\right)+(\beta+\gamma+e+h+2 \delta) d\left(\widetilde{x_{2 n+1}}, \widetilde{x_{2 n+2}}\right) \\
(1-\beta-\gamma-e-h-2 \delta) d\left(\widetilde{x_{2 n+1}}, \widetilde{x_{2 n+2}}\right) \leq(\alpha+\beta+\gamma+h+2 \delta) d\left(\widetilde{x_{2 n}}, \widetilde{x_{2 n+1}}\right) \\
d\left(\widetilde{x_{2 n+1}}, \widetilde{x_{2 n+2}}\right) \leq \frac{(\alpha+\beta+\gamma+h+2 \delta)}{(1-\beta-\gamma-e-h-2 \delta)} d\left(\widetilde{x_{2 n}}, \widetilde{x_{2 n+1}}\right)
\end{gathered}
$$

Simillaraly we can show that

$$
d\left(\widetilde{x_{2 n}}, \widetilde{x_{2 n+1}}\right) \leq \frac{(\alpha+\beta+\gamma+h+2 \delta)}{(1-\beta-\gamma-e-h-2 \delta)} d\left(\widetilde{x_{2 n-1}}, \widetilde{x_{2 n}}\right)
$$

In general we can write,

$$
d\left(\widetilde{x_{2 n+1}}, \widetilde{x_{2 n+2}}\right)=\left[\frac{(\alpha+\beta+\gamma+h+2 \delta)}{(1-\beta-\gamma-e-h-2 \delta)}\right]^{2 n+1} d\left(\widetilde{x_{0}}, \widetilde{x_{1}}\right)
$$

On taking $\frac{(\alpha+\beta+\gamma+h+2 \delta)}{(1-\beta-\gamma-e-h-2 \delta)}=\phi_{2}$

$$
d\left(\widetilde{x_{2 n+1}}, \widetilde{x_{2 n+2}}\right) \leq \phi_{2}{ }^{2 n+1} d\left(\widetilde{x_{0}}, \widetilde{x_{1}}\right)
$$

Let, $\phi=\max \left\{\phi_{1}, \phi_{2}\right\}$

For $n \leq m$ we have

$$
\begin{gathered}
d\left(\widetilde{x_{2 n}}, \widetilde{x_{2 m}}\right) \leq d\left(\widetilde{x_{2 n}}, \widetilde{x_{2 n+1}}\right)+d\left(\widetilde{x_{2 n+1}}, \widetilde{x_{2 n+2}}\right)+\ldots \ldots \ldots . .+d\left(\widetilde{x_{2 m-1}}, \widetilde{x_{2 m}}\right) \\
\leq\left(\phi^{n}+\phi^{n+1}+\phi^{n+2}+\ldots \ldots+\phi^{m}\right) d\left(\widetilde{x_{0}}, \widetilde{x_{1}}\right) \\
d\left(\widetilde{x_{2 n}}, \widetilde{x_{2 m}}\right) \leq \frac{\phi^{n}}{1-\phi} d\left(\widetilde{x_{0}}, \widetilde{x_{1}}\right)
\end{gathered}
$$

$\left\|d\left(\widetilde{x_{2 n}}, \widetilde{x_{2 m}}\right)\right\| \leq \frac{\phi^{n}}{1-\phi} r\left\|d\left(\widetilde{x_{0}}, \widetilde{x_{1}}\right)\right\|$ as $n \rightarrow \infty$ 


$$
\lim _{n \rightarrow \infty}\left\|d\left(\widetilde{x_{2 n}}, \widetilde{x_{2 m}}\right)\right\| \rightarrow 0
$$

Hence $\left\{\widetilde{x_{n}}\right\}$ is Cauchy classification in which $\tilde{v}$ touches to $\tilde{X}$.

Hence a metric $(\tilde{X}, \mathrm{~d})$ space is complete soft cone. Therefore for $\widetilde{x_{n}} \rightarrow \tilde{v}$ as $n \rightarrow \infty, A \widetilde{x_{n}} \rightarrow$ $\tilde{v}$ and $B \widetilde{x_{2 n+1}} \rightarrow \tilde{v}$ as $n \rightarrow \infty$ then $\tilde{v}$ is invariant point of $\mathrm{A}$ and $\mathrm{B}$ in $\tilde{X}$, since $\mathrm{AB}=\mathrm{BA}$ this gives $\tilde{v}=B \tilde{v}=B A \tilde{v}=A B \tilde{v}=A \tilde{v}=\tilde{v}$.

Uniqueness:Let us suppose that $\widetilde{W}$ be a new invariant point of $\mathrm{A}$ and $\mathrm{B}$ in $\tilde{X}$ which is distinct from $\widetilde{w}$, then

$B \tilde{v}=\tilde{v}$ and $B \widetilde{w}=\widetilde{w}$ also $A \tilde{v}=\tilde{v}$ and $A \widetilde{w}=\widetilde{w}$

$$
d(\tilde{v}, \widetilde{w})=d(A \tilde{v}, B \widetilde{w})
$$

From [2.1.1]

$$
\begin{aligned}
& d(A \tilde{v}, B \widetilde{w}) \leq \alpha d(\tilde{v}, \widetilde{w})+\beta[d(\tilde{v}, A \tilde{v})+d(\widetilde{w}, B \widetilde{w})]+\gamma[d(\tilde{v}, B \widetilde{w})+d(\widetilde{w}, A \tilde{v})]+ \\
& e \max \{d(\widetilde{v}, A \widetilde{v}), d(\widetilde{w}, B \widetilde{w})\}+h \max \{d(\tilde{v}, B \widetilde{w}), d(\widetilde{w}, A \widetilde{v})+ \\
& \qquad \delta\left[\frac{d(\tilde{v}, A \tilde{v})+d(\widetilde{w}, B \widetilde{w})+d(\tilde{v}, B \widetilde{w})+d(\widetilde{w}, A \tilde{v})}{1+d(\tilde{v}, A \tilde{v}) d(\widetilde{w}, B \widetilde{w}) d(\tilde{v}, B \widetilde{w}) d(\widetilde{w}, A \tilde{v})}\right] \\
& \leq \alpha d(\tilde{v}, \widetilde{w})+\beta .0+2 \gamma d(\widetilde{v}, \widetilde{w})+h \max \{d(\widetilde{v}, \widetilde{w}), d(\widetilde{w}, \tilde{v})\}+2 \delta d(\tilde{v}, \widetilde{w}) \\
& \leq \alpha d(\tilde{v}, \widetilde{w})+2 \gamma d(\tilde{v}, \widetilde{w})+h d(\tilde{v}, \widetilde{w})+2 \delta d(\tilde{v}, \widetilde{w}) \\
& d(A \tilde{v}, B \widetilde{w}) \leq(\alpha+2 \gamma+h+2 \delta) d(\tilde{v}, \widetilde{w})
\end{aligned}
$$

Which gives a contradiction. Thus $\tilde{v}$ is an communal fixed point of $\mathrm{A}$ and $\mathrm{B}$ in $\tilde{X}$.

Theorem 2.3:Let $(\tilde{X}, \mathrm{~d})$ be metric space is complete soft cone $(\mathrm{Q}, \mathrm{A})$ is regular through $\mathrm{r}$ as regular constant. Suppose that the map representing A, B and C from $\tilde{X}$ into itself satisfies the condition

$$
\begin{aligned}
& d(A B \tilde{x}, C B \tilde{y}) \leq \alpha d(\tilde{x}, \tilde{y})+\beta[d(\tilde{x}, A B \tilde{x})+d(\tilde{y}, C B \tilde{y})+\gamma[d(\tilde{x}, C B \tilde{y})+d(\tilde{y}, A B \tilde{x})] \\
&+e \max \{d(\tilde{x}, A B \tilde{x})+d(\tilde{y}, C B \tilde{y})\}+h \max \{d(\tilde{x}, C B \tilde{y}), d(\tilde{y}, A B \tilde{x})\} \\
&+\delta\left[\frac{d(\tilde{x}, A B \tilde{x})+d(\tilde{y}, C B \tilde{y})+d(\tilde{x}, C B \tilde{y})+d(\tilde{y}, A B \tilde{x})}{1+d(\tilde{x}, A B \tilde{x}) d(\tilde{y}, C B \tilde{y}) d(\tilde{x}, C B \tilde{y}) d(\tilde{y}, A B \tilde{x})}\right]
\end{aligned}
$$

For every $\tilde{x}, \tilde{y} \widetilde{\in} \tilde{X}$ and $\alpha, \beta, \gamma, e, h, \delta \geq 0$ such that $0 \leq(\beta+\gamma+h+2 \delta)+\left(\frac{\alpha+e}{2}\right)<\frac{1}{2}$. Then $\mathrm{A}, \mathrm{B}$ and $\mathrm{C}$ has communal invariant point in $\tilde{X}$. Furthermore either $\mathrm{AB}=\mathrm{BA}$ or $\mathrm{CB}=\mathrm{BC}$ then it has communal invariant point in $\tilde{X}$.

Proof:For any chance $\widetilde{x_{0}}$ in $\tilde{X}$, we have to choose $\widetilde{x_{1}}, \widetilde{x_{2}} \in \tilde{X}$ such that $A B \widetilde{x_{0}}=\widetilde{x_{1}}$ and $C B \widetilde{x_{1}}=\widetilde{x_{2}}$ 
Also, in general we can define a classification of elements in $\mathrm{X}$ we have

$\widetilde{x_{2 n+1}}=A B \widetilde{x_{2 n}}$ and $\widetilde{x_{2 n+2}}=C B \widetilde{x_{2 n+1}}$

Now, $d\left(\widetilde{x_{2 n+1}}, \widetilde{x_{2 n+2}}\right)=d\left(A B \widetilde{x_{2 n}}, C B \widetilde{x_{2 n+1}}\right)$

From [2.2.1]

$$
\begin{aligned}
& d\left(A B \widetilde{x_{2 n}}, C B \widetilde{x_{2 n+1}}\right) \\
& \leq \alpha d\left(\widetilde{x_{2 n}}, \widetilde{x_{2 n+1}}\right)+\beta\left[d\left(\widetilde{x_{2 n}}, A B \widetilde{x_{2 n}}\right)+d\left(\widetilde{x_{2 n+1}}, C B \widetilde{x_{2 n+1}}\right)\right]+ \\
& \gamma\left[d\left(\widetilde{x_{2 n}}, C B \widetilde{x_{2 n+1}}\right)+d\left(\widetilde{x_{2 n+1}}, A B \widetilde{x_{2 n}}\right)\right]+e \max \left\{d\left(\widetilde{x_{2 n}}, A B \widetilde{x_{2 n}}\right), d\left(\widetilde{x_{2 n+1}}, C \widetilde{B x_{2 n+1}}\right)\right\} \\
& +h \max \left\{d\left(\widetilde{x_{2 n}}, C B \widetilde{x_{2 n+1}}\right), d\left(\widetilde{x_{2 n+1}}, A B \widetilde{x_{2 n}}\right)\right\}+ \\
& \delta \frac{\left[d\left(\widetilde{x_{2 n}}, A B \widetilde{x_{2 n}}\right)+d\left(\widetilde{x_{2 n+1}}, C B \widetilde{x_{2 n+1}}\right)+d\left(\widetilde{x_{2 n}}, C B \widetilde{x_{2 n+1}}\right)+d\left(\widetilde{x_{2 n+1}}, A B \widetilde{x_{2 n}}\right)\right]}{1+d\left(\widetilde{x_{2 n}}, A B \widetilde{x_{2 n}}\right) d\left(\widetilde{x_{2 n+1}}, C B \widetilde{x_{2 n+1}}\right) d\left(\widetilde{x_{2 n}}, C B \widetilde{x_{2 n+1}}\right) d\left(\widetilde{x_{2 n+1}}, A B \widetilde{x_{2 n}}\right)} \\
& d\left(\widetilde{x_{2 n+1}}, \widetilde{x_{2 n+2}}\right) \\
& \leq \alpha d\left(\widetilde{x_{2 n}}, \widetilde{x_{2 n+1}}\right)+\beta\left[d\left(\widetilde{x_{2 n}}, \widetilde{x_{2 n+1}}\right)+d\left(\widetilde{x_{2 n+1}}, \widetilde{x_{2 n+2}}\right)\right]+\gamma\left[d\left(\widetilde{x_{2 n}}, \widetilde{x_{2 n+2}}\right)\right] \\
& +e \max \left\{d\left(\widetilde{x_{2 n}}, \widetilde{x_{2 n+1}}\right), d\left(\widetilde{x_{2 n+1}}, \widetilde{x_{2 n+2}}\right)\right\}+h\left\{d\left(\widetilde{x_{2 n}}, \widetilde{x_{2 n+2}}\right)\right\} \\
& +\delta \frac{\left[d\left(\widetilde{x_{2 n}}, \widetilde{x_{2 n+1}}\right)+d\left(\widetilde{x_{2 n+1}}, \widetilde{x_{2 n+2}}\right)+d\left(\widetilde{x_{2 n},} \widetilde{x_{2 n+2}}\right)+d\left(\widetilde{x_{2 n+1}}, \widetilde{x_{2 n+1}}\right)\right]}{1+d\left(\widetilde{x_{2 n}}, \widetilde{x_{2 n+1}}\right) d\left(\widetilde{x_{2 n+1}, \widetilde{x_{2 n+2}}}\right) d\left(\widetilde{x_{2 n},}, \widetilde{x_{2 n+2}}\right) d\left(\widetilde{x_{2 n+1},} \widetilde{x_{2 n+1}}\right)}
\end{aligned}
$$

\section{Consider case I:}

Let $d\left(\widetilde{x_{2 n}}, \widetilde{x_{2 n+1}}\right)>d\left(\widetilde{x_{2 n+1}}, \widetilde{x_{2 n+2}}\right)$

So, $\max \left\{d\left(\widetilde{x_{2 n}}, \widetilde{x_{2 n+1}}\right), d\left(\widetilde{x_{2 n+1}}, \widetilde{x_{2 n+2}}\right)\right\}=d\left(\widetilde{x_{2 n}}, \widetilde{x_{2 n+1}}\right)$

$$
d\left(\widetilde{x_{2 n+1}}, \widetilde{x_{2 n+2}}\right)
$$

$\leq \alpha d\left(\widetilde{x_{2 n}}, \widetilde{x_{2 n+1}}\right)+\beta\left[d\left(\widetilde{x_{2 n}}, \widetilde{x_{2 n+1}}\right)+d\left(\widetilde{x_{2 n+1}}, \widetilde{x_{2 n+2}}\right)\right]+\gamma\left[d\left(\widetilde{x_{2 n}}, \widetilde{x_{2 n+2}}\right)\right]$

$+e d\left(\widetilde{x_{2 n}}, \widetilde{x_{2 n+1}}\right)+h \max \left\{d\left(\widetilde{x_{2 n}}, \widetilde{x_{2 n+2}}\right)\right\}+2 \delta\left[d\left(\widetilde{x_{2 n}}, \widetilde{x_{2 n+1}}\right)+d\left(\widetilde{x_{2 n+1}}, \widetilde{x_{2 n+2}}\right)\right]$

$$
\begin{gathered}
\leq(\alpha+\beta+\gamma+e+h+2 \delta) d\left(\widetilde{x_{2 n}}, \widetilde{x_{2 n+1}}\right)+(\beta+\gamma+h+2 \delta) d\left(\widetilde{x_{2 n+1}}, \widetilde{x_{2 n+2}}\right) \\
(1-\beta-\gamma-h-2 \delta) d\left(\widetilde{x_{2 n+1}}, \widetilde{x_{2 n+2}}\right) \leq(\alpha+\beta+\gamma+e+h+2 \delta) d\left(\widetilde{x_{2 n}}, \widetilde{x_{2 n+1}}\right) \\
d\left(\widetilde{x_{2 n+1}}, \widetilde{x_{2 n+2}}\right) \leq \frac{(\alpha+\beta+\gamma+e+h+2 \delta)}{(1-\beta-\gamma-h-2 \delta)} d\left(\widetilde{x_{2 n}}, \widetilde{x_{2 n+1}}\right)
\end{gathered}
$$

Similarly we can show that

$$
d\left(\widetilde{x_{2 n}}, \widetilde{x_{2 n+1}}\right) \leq \frac{(\alpha+\beta+\gamma+e+h+2 \delta)}{(1-\beta-\gamma-h-2 \delta)} d\left(\widetilde{x_{2 n-1}}, \widetilde{x_{2 n}}\right)
$$

In general we can write,

$$
d\left(\widetilde{x_{2 n+1}}, \widetilde{x_{2 n+2}}\right)=\left[\frac{(\alpha+\beta+\gamma+e+h+2 \delta)}{(1-\beta-\gamma-h-2 \delta)}\right]^{2 n+1} d\left(\widetilde{x_{0}}, \widetilde{x_{1}}\right)
$$


On taking $\frac{(\alpha+\beta+\gamma+e+h+2 \delta)}{(1-\beta-\gamma-h-2 \delta)}=\phi_{1}$

$$
d\left(\widetilde{x_{2 n+1}}, \widetilde{x_{2 n+2}}\right) \leq \phi_{1}{ }^{2 n+1} d\left(\widetilde{x_{0}}, \widetilde{x_{1}}\right)
$$

\section{Case II:}

Let $d\left(\widetilde{x_{2 n+1}}, \widetilde{x_{2 n+2}}\right)>d\left(\widetilde{x_{2 n}}, \widetilde{x_{2 n+1}}\right)$

$$
\begin{gathered}
\text { So, } \max \left\{d\left(\widetilde{x_{2 n}}, \widetilde{x_{2 n+1}}\right), d\left(\widetilde{x_{2 n+1}}, \widetilde{x_{2 n+2}}\right)\right\}=d\left(\widetilde{x_{2 n+1}}, \widetilde{x_{2 n+2}}\right) \\
d\left(\widetilde{x_{2 n+1}}, \widetilde{x_{2 n+2}}\right) \\
\leq \alpha d\left(\widetilde{x_{2 n}}, \widetilde{x_{2 n+1}}\right)+\beta\left[d\left(\widetilde{x_{2 n}}, \widetilde{x_{2 n+1}}\right)+d\left(\widetilde{x_{2 n+1}}, \widetilde{x_{2 n+2}}\right)\right]+\gamma\left[d\left(\widetilde{x_{2 n}}, \widetilde{x_{2 n+2}}\right)\right] \\
+e d\left(\widetilde{x_{2 n+1}}, \widetilde{x_{2 n+2}}\right)+h \max \left\{d\left(\widetilde{x_{2 n}}, \widetilde{x_{2 n+2}}\right)\right\}+2 \delta\left[d\left(\widetilde{x_{2 n}}, \widetilde{x_{2 n+1}}\right)+d\left(\widetilde{x_{2 n+1}}, \widetilde{x_{2 n+2}}\right)\right] \\
\leq(\alpha+\beta+\gamma+h+2 \delta) d\left(\widetilde{x_{2 n}}, \widetilde{x_{2 n+1}}\right)+(\beta+\gamma+e+h+2 \delta) d\left(\widetilde{x_{2 n+1}}, \widetilde{x_{2 n+2}}\right) \\
(1-\beta-\gamma-e-h-2 \delta) d\left(\widetilde{x_{2 n+1}}, \widetilde{x_{2 n+2}}\right) \leq(\alpha+\beta+\gamma+h+2 \delta) d\left(\widetilde{x_{2 n}}, \widetilde{x_{2 n+1}}\right) \\
d\left(\widetilde{x_{2 n+1}}, \widetilde{x_{2 n+2}}\right) \leq \frac{(\alpha+\beta+\gamma+h+2 \delta)}{(1-\beta-\gamma-e-h-2 \delta)} d\left(\widetilde{x_{2 n}}, \widetilde{x_{2 n+1}}\right)
\end{gathered}
$$

Simillaraly we can show that

$$
d\left(\widetilde{x_{2 n}}, \widetilde{x_{2 n+1}}\right) \leq \frac{(\alpha+\beta+\gamma+h+2 \delta)}{(1-\beta-\gamma-e-h-2 \delta)} d\left(\widetilde{x_{2 n-1}}, \widetilde{x_{2 n}}\right)
$$

In general we can write,

$$
d\left(\widetilde{x_{2 n+1}}, \widetilde{x_{2 n+2}}\right)=\left[\frac{(\alpha+\beta+\gamma+h+2 \delta)}{(1-\beta-\gamma-e-h-2 \delta)}\right]^{2 n+1} d\left(\widetilde{x_{0}}, \widetilde{x_{1}}\right)
$$

On taking $\frac{(\alpha+\beta+\gamma+h+2 \delta)}{(1-\beta-\gamma-e-h-2 \delta)}=\phi_{2}$

$$
d\left(\widetilde{x_{2 n+1}}, \widetilde{x_{2 n+2}}\right) \leq \phi_{2}{ }^{2 n+1} d\left(\widetilde{x_{0}}, \widetilde{x_{1}}\right)
$$

Let, $\phi=\max \left\{\phi_{1}, \phi_{2}\right\}$

For $n \leq m$ we have

$$
\begin{gathered}
d\left(\widetilde{x_{2 n}}, \widetilde{x_{2 m}}\right) \leq d\left(\widetilde{x_{2 n}}, \widetilde{x_{2 n+1}}\right)+d\left(\widetilde{x_{2 n+1}}, \widetilde{x_{2 n+2}}\right)+\ldots \ldots \ldots . .+d\left(\widetilde{x_{2 m-1}}, \widetilde{x_{2 m}}\right) \\
\leq\left(\phi^{n}+\phi^{n+1}+\phi^{n+2}+\ldots \ldots+\phi^{m}\right) d\left(\widetilde{x_{0}}, \widetilde{x_{1}}\right) \\
d\left(\widetilde{x_{2 n}}, \widetilde{x_{2 m}}\right) \leq \frac{\phi^{n}}{1-\phi} d\left(\widetilde{x_{0}}, \widetilde{x_{1}}\right)
\end{gathered}
$$

$\left\|d\left(\widetilde{x_{2 n}}, \widetilde{x_{2 m}}\right)\right\| \leq \frac{\phi^{n}}{1-\phi} r\left\|d\left(\widetilde{x_{0}}, \widetilde{x_{1}}\right)\right\|$ as $n \rightarrow \infty$

$$
\lim _{n \rightarrow \infty}\left\|d\left(\widetilde{x_{2 n}}, \widetilde{x_{2 m}}\right)\right\| \rightarrow 0
$$

Hence $\left\{\widetilde{x_{n}}\right\}$ is Cauchy classification in which $\tilde{v}$ touches to $\tilde{X}$. 
Hence a metric $(\tilde{X}, \mathrm{~d})$ space is complete soft cone. Therefore for $\widetilde{x_{n}} \rightarrow \tilde{v}$ as $n \rightarrow \infty, A B \widetilde{x_{n}} \rightarrow$ $\tilde{v}$ and $C B \widetilde{x_{2 n+1}} \rightarrow \tilde{v}$ as $n \rightarrow \infty$ then $\tilde{v}$ is invariant point of $\mathrm{A}$ and $\mathrm{C}$ in $\tilde{X}$, since $\mathrm{AC}=\mathrm{CA}$ this gives $\tilde{v}=C \tilde{v}=C A \tilde{v}=A C \tilde{v}=A \tilde{v}=\tilde{v}$.

$\tilde{v}$ is communal invariant point of $\mathrm{A}$ and $\mathrm{C}$.

Uniqueness:Let us suppose that $\widetilde{W}$ be a new invariant point of $\mathrm{A}$ and $\mathrm{B}$ in $\tilde{X}$ which is distinct from $\widetilde{w}$, then

$C \tilde{v}=\tilde{v}$ and $C \widetilde{w}=\widetilde{w}$ also $A \tilde{v}=\tilde{v}$ and $A \widetilde{w}=\widetilde{w}$

$$
d(\tilde{v}, \widetilde{w})=d(A \tilde{v}, C \widetilde{w})
$$

From [2.1.1]

$$
\begin{aligned}
& d(A \tilde{v}, C \widetilde{w}) \leq \alpha d(\tilde{v}, \widetilde{w})+\beta[d(\tilde{v}, A \tilde{v})+d(\widetilde{w}, C \widetilde{w})]+\gamma[d(\tilde{v}, C \widetilde{w})+d(\widetilde{w}, A \tilde{v})]+ \\
& e \max \{d(\tilde{v}, A \tilde{v}), d(\widetilde{w}, C \widetilde{w})\}+h \max \{d(\tilde{v}, C \widetilde{w}), d(\widetilde{w}, A \widetilde{v})+ \\
& \delta\left[\frac{d(\tilde{v}, A \tilde{v})+d(\widetilde{w}, C \widetilde{w})+d(\tilde{v}, C \widetilde{w})+d(\widetilde{w}, A \tilde{v})}{1+d(\tilde{v}, A \tilde{v}) d(\widetilde{w}, C \widetilde{w}) d(\tilde{v}, C \widetilde{w}) d(\widetilde{w}, A \tilde{v})}\right] \\
& \leq \alpha d(\tilde{v}, \widetilde{w})+\beta .0+2 \gamma d(\tilde{v}, \widetilde{w})+h \max \{d(\tilde{v}, \widetilde{w}), d(\widetilde{w}, \tilde{v})\}+2 \delta d(\tilde{v}, \widetilde{w}) \\
& \leq \alpha d(\tilde{v}, \widetilde{w})+2 \gamma d(\tilde{v}, \widetilde{w})+h d(\tilde{v}, \widetilde{w})+2 \delta d(\tilde{v}, \widetilde{w}) \\
& d(A \tilde{v}, C \widetilde{w}) \leq(\alpha+2 \gamma+h+2 \delta) d(\tilde{v}, \widetilde{w})
\end{aligned}
$$

Which gives a contradiction. Thus $\tilde{v}$ is an communal fixed point of $\mathrm{A}$ and $\mathrm{C}$ in $\tilde{X}$.

Theorem 2.4:Let the metric space $(\tilde{X}, \mathrm{~d})$ be with complete soft cone has $(\mathrm{Q}, \mathbb{A})$ is regular through $r$ as regular constant. Let the map representing $\mathrm{G}, \mathrm{H}, \mathrm{A}$ and $\mathrm{B}$ from $\tilde{X}$ into itself satisfies the particular condition as:

i) $\mathrm{G}(\tilde{X}) \subseteq \mathrm{B}(\tilde{X}), \mathrm{H}(\tilde{X}) \subseteq \mathrm{A}(\tilde{X})$

ii) $[\mathrm{G}, \mathrm{A}]$ and $[\mathrm{H}, \mathrm{B}]$ are inadequately compatible

iii) A or B is unceasing

iv) $d(G \tilde{x}, H \tilde{y}) \leq \alpha d(A \tilde{x}, B \tilde{y})+\beta[d(\widetilde{A x}, G \tilde{x})+d(B \tilde{y}, H \tilde{y})+\gamma[d(A \tilde{x}, H \tilde{y})+d(B \tilde{y}, G \tilde{x})]$

$+e \max \{d(\widetilde{A x}, G \tilde{x})+d(B \tilde{y}, H \tilde{y})\}+h \max \{d(A \tilde{x}, H \tilde{y}), d(B \tilde{y}, G \tilde{x})\}$

$$
+\delta\left[\frac{d(A \tilde{x}, G \tilde{x})+d(\widetilde{B y}, H \tilde{y})+d(A \tilde{x}, H \tilde{y})+d(B \tilde{y}, G \tilde{x})}{1+d(A \tilde{x}, G \tilde{x}) d(B \tilde{y}, H \tilde{y}) d(A \tilde{x}, H \tilde{y}) d(B \tilde{y}, G \tilde{x})}\right]
$$

For every $\tilde{x}, \tilde{y} \widetilde{\in} \tilde{X}$ and $\alpha, \beta, \gamma, e, h, \delta \geq 0$ such that $0 \leq(\beta+\gamma+h+2 \delta)+\left(\frac{\alpha+e}{2}\right)<\frac{1}{2}$. Then $\mathrm{G}, \mathrm{H}, \mathrm{A}$ and $\mathrm{B}$ has communal invariant point in $\tilde{X}$. 
Proof:Let us designate a sequence $\left\{\widetilde{x_{n}}\right\}$ and $\left\{\widetilde{y_{n}}\right\}$ in $\tilde{X}$ such that

$G \widetilde{x_{2 n}}=B \widetilde{x_{2 n+1}}=\widetilde{y_{2 n}}$ and $\mathrm{H} \widetilde{x_{2 n+1}}=A \widetilde{x_{2 n+2}}=\widetilde{y_{2 n+2}}, \forall n=0,1,2, \ldots \ldots$

Now, $d\left(\widetilde{y_{2 n}}, \widetilde{y_{2 n+1}}\right)=d\left(\widetilde{x_{2 n}}, H \widetilde{x_{2 n+1}}\right)$

From [2.4.1]

$$
d\left(G \widetilde{x_{2 n}}, H \widetilde{x_{2 n+1}}\right)
$$

$\leq \alpha d\left(\widetilde{A x_{2 n}}, B \widetilde{x_{2 n+1}}\right)+\beta\left[d\left(\widetilde{A x_{2 n}}, G \widetilde{x_{2 n}}\right)+d\left(\widetilde{A x_{2 n+1}}, H \widetilde{x_{2 n+1}}\right)\right]+$

$$
\gamma\left[d\left(A \widetilde{x_{2 n}}, H \widetilde{x_{2 n+1}}\right)+d\left(B \widetilde{x_{2 n+1}}, G \widetilde{x_{2 n}}\right)\right]+e \max \left\{d\left(A \widetilde{x_{2 n}}, G \widetilde{x_{2 n}}\right), d\left(\widetilde{B x_{2 n+1}}, H \widetilde{x_{2 n+1}}\right)\right\}
$$

$+h \max \left\{d\left(\widetilde{A x_{2 n}}, H \widetilde{x_{2 n+1}}\right), d\left(B \widetilde{x_{2 n+1}}, G \widetilde{x_{2 n}}\right)\right\}+$

$$
\begin{aligned}
& \delta \frac{\left[d\left(A \widetilde{x_{2 n}}, G \widetilde{x_{2 n}}\right)+d\left(B \widetilde{x_{2 n+1}}, H \widetilde{x_{2 n+1}}\right)+d\left(A \widetilde{x_{2 n}}, H \widetilde{x_{2 n+1}}\right)+d\left(\widetilde{x_{2 n+1}}, G \widetilde{x_{2 n}}\right)\right]}{1+d\left(A \widetilde{x_{2 n}}, G \widetilde{x_{2 n}}\right) d\left(B \widetilde{x_{2 n+1}}, H \widetilde{x_{2 n+1}}\right) d\left(A \widetilde{x_{2 n}}, H \widetilde{x_{2 n+1}}\right) d\left(B \widetilde{x_{2 n+1}}, G \widetilde{x_{2 n}}\right)} \\
& d\left(\widetilde{y_{2 n}}, \widetilde{y_{2 n+1}}\right) \\
& \leq \alpha d\left(\widetilde{y_{2 n-1}}, \widetilde{y_{2 n}}\right)+\beta\left[d\left(\widetilde{y_{2 n-1}}, \widetilde{y_{2 n}}\right)+d\left(\widetilde{y_{2 n}}, \widetilde{y_{2 n+1}}\right)\right]+\gamma\left[d\left(\widetilde{y_{2 n-1}}, \widetilde{y_{2 n+1}}\right)\right] \\
& +e \max \left\{d\left(\widetilde{y_{2 n-1}}, \widetilde{y_{2 n}}\right), d\left(\widetilde{y_{2 n}}, \widetilde{y_{2 n+1}}\right)\right\}+h\left\{d\left(\widetilde{y_{2 n-1}}, \widetilde{y_{2 n+1}}\right)\right\} \\
& +\delta \frac{\left[d\left(\widetilde{y_{2 n-1}}, \widetilde{y_{2 n}}\right)+d\left(\widetilde{y_{2 n}}, \widetilde{y_{2 n+1}}\right)+d\left(\widetilde{y_{2 n-1}}, \widetilde{y_{2 n+1}}\right)+d\left(\widetilde{y_{2 n}}, \widetilde{y_{2 n}}\right)\right]}{1+d\left(\widetilde{y_{2 n-1}}, \widetilde{y_{2 n}}\right) d\left(\widetilde{y_{2 n}}, \widetilde{y_{2 n+1}}\right) d\left(\widetilde{y_{2 n-1}}, \widetilde{y_{2 n+1}}\right) d\left(\widetilde{y_{2 n}}, \widetilde{y_{2 n}}\right)}
\end{aligned}
$$

\section{Consider case I:}

Let $d\left(\widetilde{y_{2 n-1}}, \widetilde{y_{2 n}}\right)>d\left(\widetilde{y_{2 n}}, \widetilde{y_{2 n+1}}\right)$

So, $\max \left\{d\left(\widetilde{y_{2 n-1}}, \widetilde{y_{2 n}}\right), d\left(\widetilde{y_{2 n}}, \widetilde{y_{2 n+1}}\right)\right\}=d\left(\widetilde{y_{2 n-1}}, \widetilde{y_{2 n}}\right)$

$$
d\left(\widetilde{y_{2 n}}, \widetilde{y_{2 n+1}}\right)
$$

$\leq \alpha d\left(\widetilde{y_{2 n-1}}, \widetilde{y_{2 n}}\right)+\beta\left[d\left(\widetilde{y_{2 n-1}}, \widetilde{y_{2 n}}\right)+d\left(\widetilde{y_{2 n}}, \widetilde{y_{2 n+1}}\right)\right]+\gamma\left[d\left(\widetilde{y_{2 n-1}}, \widetilde{y_{2 n+1}}\right)\right]$

$+e d\left(\widetilde{y_{2 n-1}}, \widetilde{y_{2 n}}\right)+h \max \left\{d\left(\widetilde{y_{2 n-1}}, \widetilde{y_{2 n+1}}\right)\right\}+2 \delta\left[d\left(\widetilde{y_{2 n-1}}, \widetilde{y_{2 n}}\right)+d\left(\widetilde{y_{2 n}}, \widetilde{y_{2 n+1}}\right)\right]$

$$
\begin{gathered}
\leq(\alpha+\beta+\gamma+e+h+2 \delta) d\left(\widetilde{y_{2 n-1}}, \widetilde{y_{2 n}}\right)+(\beta+\gamma+h+2 \delta) d\left(\widetilde{y_{2 n}}, \widetilde{y_{2 n+1}}\right) \\
(1-\beta-\gamma-h-2 \delta) d\left(\widetilde{y_{2 n}}, \widetilde{y_{2 n+1}}\right) \leq(\alpha+\beta+\gamma+e+h+2 \delta) d\left(\widetilde{y_{2 n-1}}, \widetilde{y_{2 n}}\right) \\
d\left(\widetilde{y_{2 n}}, \widetilde{y_{2 n+1}}\right) \leq \frac{(\alpha+\beta+\gamma+e+h+2 \delta)}{(1-\beta-\gamma-h-2 \delta)} d\left(\widetilde{y_{2 n-1}}, \widetilde{y_{2 n}}\right)
\end{gathered}
$$

Similarly we can show that

$$
d\left(\widetilde{y_{2 n-1}}, \widetilde{y_{2 n}}\right) \leq \frac{(\alpha+\beta+\gamma+e+h+2 \delta)}{(1-\beta-\gamma-h-2 \delta)} d\left(\widetilde{y_{2 n-2}}, \widetilde{y_{2 n-1}}\right)
$$

In general we can write,

$$
d\left(\widetilde{y_{2 n}}, \widetilde{y_{2 n+1}}\right)=\left[\frac{(\alpha+\beta+\gamma+e+h+2 \delta)}{(1-\beta-\gamma-h-2 \delta)}\right]^{2 n+1} d\left(\widetilde{y_{0}}, \widetilde{y_{1}}\right)
$$


On taking $\frac{(\alpha+\beta+\gamma+e+h+2 \delta)}{(1-\beta-\gamma-h-2 \delta)}=\phi_{1}$

$$
d\left(\widetilde{y_{2 n}}, \widetilde{y_{2 n+1}}\right) \leq \phi_{1}{ }^{2 n+1} d\left(\widetilde{y_{0}}, \widetilde{y_{1}}\right)
$$

\section{Case II:}

Let $d\left(\widetilde{y_{2 n}}, \widetilde{y_{2 n+1}}\right)>d\left(\widetilde{y_{2 n-1}}, \widetilde{y_{2 n}}\right)$

$$
\begin{gathered}
\text { So, } \max \left\{d\left(\widetilde{y_{2 n-1}}, \widetilde{y_{2 n}}\right), d\left(\widetilde{y_{2 n}}, \widetilde{y_{2 n+1}}\right)\right\}=d\left(\widetilde{y_{2 n}}, \widetilde{y_{2 n+1}}\right) \\
d\left(\widetilde{y_{2 n}}, \widetilde{y_{2 n+1}}\right) \\
\leq \alpha d\left(\widetilde{y_{2 n-1}}, \widetilde{y_{2 n}}\right)+\beta\left[d\left(\widetilde{y_{2 n-1}}, \widetilde{y_{2 n}}\right)+d\left(\widetilde{y_{2 n}}, \widetilde{y_{2 n+1}}\right)\right]+\gamma\left[d\left(\widetilde{y_{2 n-1}}, \widetilde{y_{2 n+1}}\right)\right] \\
+e d\left(\widetilde{y_{2 n}}, \widetilde{y_{2 n+1}}\right)+h d\left(\widetilde{y_{2 n-1}}, \widetilde{y_{2 n+1}}\right)+2 \delta\left[d\left(\widetilde{y_{2 n-1}}, \widetilde{y_{2 n}}\right)+d\left(\widetilde{y_{2 n}}, \widetilde{y_{2 n+1}}\right)\right] \\
\leq(\alpha+\beta+\gamma+h+2 \delta) d\left(\widetilde{y_{2 n-1}}, \widetilde{y_{2 n}}\right)+(\beta+\gamma+h+e+2 \delta) d\left(\widetilde{y_{2 n}}, \widetilde{y_{2 n+1}}\right) \\
(1-\beta-\gamma-e-h-2 \delta) d\left(\widetilde{y_{2 n}}, \widetilde{y_{2 n+1}}\right) \leq(\alpha+\beta+\gamma+h+2 \delta) d\left(\widetilde{y_{2 n-1}}, \widetilde{y_{2 n}}\right) \\
d\left(\widetilde{y_{2 n}}, \widetilde{y_{2 n+1}}\right) \leq \frac{(\alpha+\beta+\gamma+h+2 \delta)}{(1-\beta-\gamma-e-h-2 \delta)} d\left(\widetilde{y_{2 n-1}}, \widetilde{y_{2 n}}\right)
\end{gathered}
$$

Similarly we can show that

$$
d\left(\widetilde{y_{2 n-1}}, \widetilde{y_{2 n}}\right) \leq \frac{(\alpha+\beta+\gamma+h+2 \delta)}{(1-\beta-\gamma-e-h-2 \delta)} d\left(\widetilde{y_{2 n-2}}, \widetilde{y_{2 n-1}}\right)
$$

In general we can write,

$$
d\left(\widetilde{y_{2 n}}, \widetilde{y_{2 n+1}}\right)=\left[\frac{(\alpha+\beta+\gamma+h+2 \delta)}{(1-\beta-\gamma-e-h-2 \delta)}\right]^{2 n+1} d\left(\widetilde{y_{0}}, \widetilde{y_{1}}\right)
$$

On taking $\frac{(\alpha+\beta+\gamma+h+2 \delta)}{(1-\beta-\gamma-e-h-2 \delta)}=\phi_{2}$

$$
d\left(\widetilde{y_{2 n}}, \widetilde{y_{2 n+1}}\right) \leq \phi_{2}{ }^{2 n+1} d\left(\widetilde{y_{0}}, \widetilde{y_{1}}\right)
$$

Let, $\phi=\max \left\{\phi_{1}, \phi_{2}\right\}$

For $n \leq m$ we have

$$
\begin{gathered}
\left.d\left(\widetilde{y_{2 n}}, \widetilde{y_{2 m}}\right) \leq d\left(\widetilde{y_{2 n}}, \widetilde{y_{2 n+1}}\right)+d\left(\widetilde{y_{2 n+1}}, \widetilde{y_{2 n+2}}\right)+\ldots \ldots \ldots . .+\widetilde{y_{2 m-1}}, \widetilde{y_{2 m}}\right) \\
\leq\left(\phi^{n}+\phi^{n+1}+\phi^{n+2}+\ldots \ldots+\phi^{m}\right) d\left(\widetilde{y_{0}}, \widetilde{y_{1}}\right) \\
d\left(\widetilde{y_{2 n}}, \widetilde{y_{2 m}}\right) \leq \frac{\phi^{n}}{1-\phi} d\left(\widetilde{y_{0}}, \widetilde{y_{1}}\right)
\end{gathered}
$$

$\left\|d\left(\widetilde{y_{2 n}}, \widetilde{y_{2 m}}\right)\right\| \leq \frac{\phi^{n}}{1-\phi} r\left\|d\left(\widetilde{y_{0}}, \widetilde{y_{1}}\right)\right\|$ as $n \rightarrow \infty$

$$
\lim _{n \rightarrow \infty}\left\|d\left(\widetilde{y_{2 n}}, \widetilde{y_{2 m}}\right)\right\| \rightarrow 0
$$

Hence $\left\{\widetilde{y_{n}}\right\}$ is Cauchy classification in which $\tilde{v}$ touches to $\tilde{X}$ by stability of A and B. 
Correspondingly the classification $\left\{\widetilde{y_{n}}\right\}$ is also convergent classification in which $\tilde{v}$ touches to $\tilde{X}$

Hence a metric space $(\tilde{X}, \mathrm{~d})$ is complete soft cone and $\tilde{v}$ is an invariant point of $\mathrm{G}, \mathrm{H}, \mathrm{A}$ and $\mathrm{B}$. Since $\{\mathrm{G}, \mathrm{A}\}$ and $\{\mathrm{H}, \mathrm{B}\}$ are inadequately compatible which gives that $\tilde{v}$ is communal invariant point of $\mathrm{G}, \mathrm{H}, \mathrm{A}$ and $\mathrm{B}$.

Uniqueness:Let us suppose that $\tilde{z}$ be a new invariant point of G, H, A and B in $\tilde{X}$ which is distinct from $\tilde{v}$, then

$G \tilde{v}=\tilde{v}$ and $G \tilde{z}=\tilde{z}$ also $H \tilde{v}=\tilde{v}$ and $H \tilde{z}=\tilde{z}$

$$
d(\tilde{v}, \widetilde{w})=d(G \tilde{v}, H \tilde{z})
$$

From [2.4.1]

$$
\begin{aligned}
& d(G \tilde{v}, H \tilde{z}) \leq \alpha d(A \tilde{v}, B \tilde{z})+\beta[d(A \tilde{v}, G \tilde{v})+d(B \tilde{z}, H \tilde{z})]+\gamma[d(A \tilde{v}, H \tilde{z})+d(B \tilde{z}, G \tilde{v})]+ \\
& e \max \{d(\tilde{A v}, G \tilde{v}), d(B \tilde{z}, H \tilde{z})\}+h \max \{d(A \tilde{v}, H \tilde{z}), d(B \tilde{z}, G \tilde{v})+ \\
& \qquad \delta\left[\frac{d(A \tilde{v}, G \tilde{v})+d(B \tilde{z}, H \tilde{z})+d(A \tilde{v}, H \tilde{z})+d(B \tilde{z}, G \tilde{v})}{1+d(A \tilde{v}, G \tilde{v}) d(B \tilde{z}, H \tilde{z}) d(A \tilde{v}, H \tilde{z}) d(B \tilde{z}, G \tilde{v})}\right] \\
& \leq \alpha d(\tilde{v}, \tilde{z})+2 \gamma d(\tilde{v}, \tilde{z})+h d(\tilde{v}, \tilde{z})+2 \delta d(\tilde{v}, \tilde{z}) \\
& d(G \tilde{v}, H \tilde{z}) \leq(\alpha+2 \gamma+h+2 \delta) d(\tilde{v}, \tilde{z})
\end{aligned}
$$

Which gives a contradiction. Thus $\tilde{v}$ is an communal fixed point of $\mathrm{G}, \mathrm{H}, \mathrm{A}$ and $\mathrm{B}$ in $\tilde{X}$.

\section{REFERENCES:}

[1] W.L. Gau and D.J. Buehrer, vague sets, IEEE. Trains system man Cybernet 23(2), (1993), 610-614.

[2] T.Y. Lin, Granular computing on binary relation II: Rough sets representation and belief function, In rough set in knowledge discovery, (Edited by A. Skowarn and L. Polkowski), spriger-verlag (1998), 121-140.

[3] Z. Pawlak, Rough sets, International jouranal of information and computer sci.11,(1982), 341-356.

[4] L.A. Zadeh, fuzzy sets, Infor. And control 8,(1965), 338-353.

[5] D. Molodtsov, Soft set theory-first results, Comput. Math. Appl. 37 (4) (1999) 19-31. doi:10.1016/S08981221(99)00056-5.

[6] P. K. Maji, R. Biswas, A. R. Roy, Soft set theory, Comput. Math. Appl. 45 (4) (2003) 555-562. doi:10.1016/S0898-1221(03)00016-6.

[7] P. K. Maji, A. R. Roy, R. Biswas, An application of soft sets in a decision making problem, Comput. Math. Appl. 44 (8) (2002) 1077-1083. doi:10.1016/S0898-1221(02)00216-X.

[8] D. Chen, E. C. C. Tsang, D. S. Yeung, X. Wang, The parameterization reduction of soft sets and its applications, Comput. Math. Appl. 49 (5) (2005) 757-763. doi:10.1016/j.camwa.2004.10.036.

[9] D. Pei, D. Miao, From soft sets to information systems, in: 2005 IEEE International Conference on Granular Computing, Vol. 2, IEEE, 2005, pp. 617-621. 
[10] Z. Kong, W. Jia, G. Zhang, L. Wang, Normal parameter reduction in soft set based on particle swarm optimization algorithm, Appl. Math. Model. 39 (16) (2015) 4808-4820.

[11] Y. Zou, Z. Xiao, Data analysis approaches of soft sets under incomplete information, Knowledge-Based Systems 21 (8) (2008) 941-945.

[12] H. Aktas, N. Cagman, Soft sets and soft groups, Inform. Sci. 177 (13) (2007) 2726-2735.

[13] M. Shabir, M. Naz, On soft topological spaces, Comput. Math. Appl. 61 (7) (2011) 1786 - 1799.

[14] A. Aygüno־glu, H. Aygu“n, Some notes on soft topological spaces, Neural Computing and Applications 21 (1) (2012) 113-119.

[15] V. Cetkin, H. Aygu“n, On convergence of soft nets, J. Mult.-Valued Logic Soft Comput. 26 (3-5) (2016) $175-187$.

[16] S. Das, S. K. Samanta, Soft real sets, soft real numbers and their properties, J. Fuzzy Math. 20 (3) (2012) $551-576$.

[17] S. Das, S. K. Samanta, On soft complex sets and soft complex numbers, J. Fuzzy Math. 21 (1) (2013) 195216.

[18] S. Das, S. K. Samanta, On soft metric spaces, J. Fuzzy Math. 21 (3) (2013) 707-734.

[19] S. Das, S. K. Samanta, Soft metric, Ann. Fuzzy Math. Inform. 6 (1) (2013) 77-94.

[20] S. Das, S. K. Samanta, On soft inner product spaces, Ann. Fuzzy Math. Inform. 6 (1) (2013) 151-170.

[21] S. Das, S. K. Samanta, Soft linear operators in soft normed linear spaces, Ann. Fuzzy Math. Inform. 6 (2) (2013) 295-314.

[22] M. Chiney, S. K. Samanta, Vector soft topology, Ann. Fuzzy Math. Inform. 10 (1) (2015) $45-64$.

[23] S. Das, P. Majumdar, S. K. Samanta, On soft linear spaces and soft normed linear spaces, Ann. Fuzzy Math. Inform. 9 (1) (2015) 91-109.

[24] A. C. Guler, E. D. Yildirim, O. B. Ozbakir, A fixed point theorem on soft G-metric spaces, J. Nonlinear Sci. Appl. 9 (3) (2016) 885-894.

[25] L.-G. Huang, X. Zhang, Cone metric spaces and fixed point theorems of contractive mappings, J. Math. Anal. Appl. 332 (2) (2007) 1468-1476.

[26] S. Rezapour, R. Hamlbarani, Some notes on the paper Cone metric spaces and fixed point theorems of contractive mappings, J. Math. Anal. Appl. 345 (2) (2008) 719-724.

[27] N. Mehmood, A. Azam, L. D. R. Ko`cinac, Multivalued fixed point results in cone metric spaces, Topology Appl. 179 (2015) 156-170.

[28] M. Abbas, B. Ali, C. Vetro, A suzuki type fixed point theorem for a generalized multivalued mapping on partial Hausdorff metric spaces, Topology Appl. 160 (3) (2013) 553-563.

[29] M. Abbas, G. Jungck, Common fixed point results for noncommuting mappings without continuity in cone metric spaces, J. Math. Anal. Appl. 341 (1) (2008) 416-420.

[30] I. Altun, "O. Acar, Fixed point theorems for weak contractions in the sense of Berinde on partial metric spaces, Topology Appl. 159 (10) (2012) 2642-2648.

[31] Ismet A. and Kemal T., soft cone metric spaces and some fixed point theorem, arxiv:1610.01515v1 [Math.GM](2016)

[32] M. MOhammad, R. Jamal, R. Bharadwaj and Q.A. Kabir, International journal of Mathematical Archive8(9),(2017), 11-16. 